\title{
Recent Studies on Inverse Medium Scattering Problems
}

\author{
Gang BaO*, Peijun Li ${ }^{\dagger}$, And Songming Hou ${ }^{\ddagger}$
}

\begin{abstract}
Regularized recursive linearization methods are presented for solving a two-dimensional inverse medium scattering problem, which reconstructs the scatterer of an inhomogeneous medium from the measurements of near field current densities. Energy estimates of the scattered field are obtained on which the Born approximation is based. The methods start from the Born approximation corresponding to the weak scattering, each update is obtained via recursive linearization with respect to the wavenumber or the spatial frequency by solving one forward problem and one adjoint problem of the Helmholtz equation. In the case that the weak scattering criterion is not satisfied, a technique based on a direct imaging algorithm may be developed to generate an initial guess. Numerical examples are presented to illustrate the efficiency and robustness of the underlying computational method.
\end{abstract}

\section{Introduction}

Consider the Helmholtz equation in two dimensions,

$$
\Delta \phi(\mathbf{x})+\kappa^{2}(1+q(\mathbf{x})) \phi(\mathbf{x})=0, \quad \mathbf{x} \in \mathbb{R}^{2},
$$

where $\phi$ is the total field, $\kappa$ is a positive real number, known as the wavenumber, and the scatterer $q$ is a function with $q(\mathbf{x})>-1$ for all $\mathbf{x} \in \mathbb{R}^{2}$. The function $q$ is assumed to be supported in a bounded domain $\Omega \subset \mathbb{R}^{2}$, as shown in Figure 1 .

Denote the wave vector $\mathbf{k}=(\eta, k(\eta))$, where $\eta$ is the transverse part of the wave vector and

$$
k(\eta)= \begin{cases}\sqrt{\kappa^{2}-\eta^{2}}, & \text { for } \kappa>|\eta|, \\ \mathrm{i} \sqrt{\eta^{2}-\kappa^{2}}, & \text { for } \kappa<|\eta| .\end{cases}
$$

The number $|\eta|$ is known as the spatial frequency.

The scatterer is illuminated by a one-parameter family of plane waves

$$
\phi_{0}(\mathbf{x})=e^{\mathrm{ik} \cdot \mathbf{x}} .
$$

\footnotetext{
*Department of Mathematics, Michigan State University, East Lansing, MI 48824 (bao@math.msu.edu). The research was supported in part by the NSF grants DMS 01-04001 and CCF-0514078, the ONR grant N000140210365, the National Science Foundation of China grant 10428105.

${ }^{\dagger}$ Department of Mathematics, University of Michigan, Ann Arbor, MI 48109 (lipeijun@umich.edu).

${ }^{\ddagger}$ Department of Mathematics, Michigan State University, East Lansing, MI 48824 (mickey@math.msu.edu).
} 


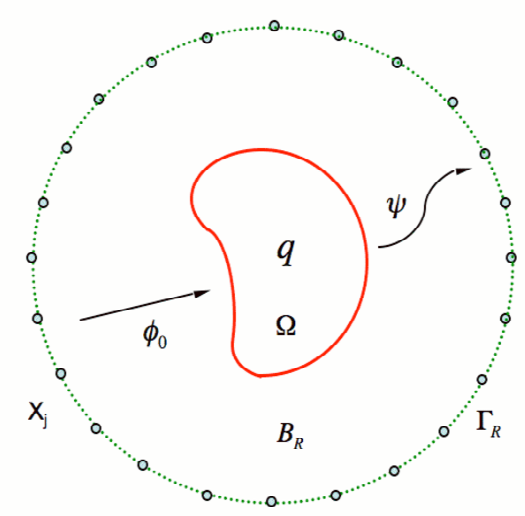

Figure 1: The problem geometry. For a plane wave $\phi_{0}$ incident on the scatterer $q$, the scattered wave $\psi$ is measured at $\mathbf{x}_{j}, j=1, \ldots, m$.

The modes for which $|\eta| \leq \kappa$ correspond to propagating plane waves while the modes with $|\eta|>\kappa$ correspond to evanescent plane waves. For propagating waves, we explicitly have by letting $\eta=\kappa \cos \alpha$ and $k(\eta)=\kappa \sin \alpha$ that

$$
\phi_{0}(\mathbf{x})=e^{\mathrm{i} \kappa \mathbf{d} \cdot \mathbf{x}} .
$$

Here $\mathbf{d}=(\cos \alpha, \sin \alpha)$ denotes the propagation direction. For evanescent waves, we explicitly have

$$
\phi_{0}(\mathbf{x})=e^{\mathrm{i} \eta x_{1}-\sqrt{\eta^{2}-\kappa^{2}} x_{2}} .
$$

These waves are oscillatory parallel to the $x_{1}$ axis and decay exponentially along the $x_{2}$ axis. The higher the spatial frequency of the evanescent plane waves used to probe the scatterer is, the more rapidly the field decays as a function of depth into the scatterer. It is well known that the high spatial frequency evanescent plane waves may be generated at the interface of two media by total internal reflection [11,16], which has been in practical use for decades, especially in near-field optics [24]. A recent review on the near-field optics and near-field microscopy may be found in [20]. Evidently, such incident waves satisfy the homogeneous equation

$$
\Delta \phi_{0}+\kappa^{2} \phi_{0}=0 .
$$

The total field $\phi$ takes the form

$$
\phi=\phi_{0}+\psi
$$

Here $\psi: \mathbb{R}^{2} \mapsto \mathbb{C}$ is the scattered field which satisfies from (1.1), (1.5), and (1.6) that

$$
\Delta \psi(\mathbf{x})+\kappa^{2}(1+q(\mathbf{x})) \psi(\mathbf{x})=-\kappa^{2} q(\mathbf{x}) \phi_{0}(\mathbf{x}), \quad \mathbf{x} \in \mathbb{R}^{2},
$$

and the Sommerfeld radiation condition

$$
\lim _{r \rightarrow \infty} \sqrt{r}\left(\frac{\partial \psi}{\partial r}-\mathrm{i} \kappa \psi\right)=0, \quad r=|\mathbf{x}|,
$$

uniformly along all directions $\mathbf{x} /|\mathbf{x}|$. 
In this context, the direct scattering problem is to determine the scattered field $\psi$, given the incident field $\phi_{0}$ and the scatterer $q$, which has been studied extensively over the last few decades $[18,32,35]$. To serve our general purpose, we restrict to the finite element method for solving the direct problem numerically. A crucial step is to truncate the infinite physical domain to a bounded domain around the scatterer by introducing suitable artificial boundary conditions for the truncated domain. Based on the Dirichlet-to-Neumann map, a nonlocal transparent boundary condition is adopted for the finite element method. Using the Lax-Milgram lemma and the Fredholm alternative, the direct problem is shown in this survey to have a unique solution for all but possibly a discrete set of wavenumbers. Energy estimates for the scattered field are established, which provide criteria for the weak scattering. For the regularity of the scattered field, the reader is referred to [3]. The inverse scattering problem is to reconstruct the scatterer $q$ from the measurements of the scattered field $\psi$ at $\mathbf{x}_{j}, j=1, \ldots, m$, given the incident field $\phi_{0}$. The inverse problem arises naturally in diverse applications such as radar and sonar, geophysical exploration, medical imaging, and nondestructive testing $[19,33]$. However, numerical solution of the inverse problem remains challenging for the following two prinicple reasons. The inverse problem is inherently nonlinear. From the point of view of numerical computations, the problem is also severely ill-posed. In particular, small variations in the measured data can lead to large errors in the reconstruction.

The goal of this work is to report our progress on regularized recursive linearization methods for solving the inverse problems for the Helmholtz equation with multiple and single frequency scattering data. The reader is referred to $[5-7,14,15]$ for solving the inverse problems in the two-dimensional Helmholtz equation and the three-dimensional Maxwell equations in the case of full aperture data. In the limited aperture case, the reader is referred to [8] and [9] for homogeneous and more recently inhomogeneous background medium. Finally, due to the space limitation, no attempt has been made to cover other relevant approaches. We refer the reader to $[21,26,34,39]$ for related results on the inverse medium scattering problem. See $[17,19]$ for an account of recent scattering progress on the general inverse scattering problem.

The outline of the survey is as follows. In Section 2, the variational problem for direct scattering is analyzed and energy estimates on the scattered field are given. Initial guesses of the reconstruction from the Born approximation or from a direct imaging algorithm are derived in Section 3. Regularized recursive linearization methods are presented in Section 4. Section 5 is devoted to the numerical study of the proposed methods. The survey is concluded with some general remarks and directions for future research in Section 6 .

\section{Analysis of the direct scattering}

In this section, the variational formulation for the direct problem is discussed. The analysis provides some criteria for the weak scattering, which plays an important role in the inversion method.

Let the support of the scatterer $\Omega$ be contained in the interior of the ball $B_{R}=\left\{\mathbf{x} \in \mathbb{R}^{2}:|\mathbf{x}|<R\right\}$ with boundary $\Gamma_{R}=\partial B_{R}$, as seen in Figure 1 . In the domain $\mathbb{R}^{2} \backslash \bar{B}_{R}$, the solution of (1.7), (1.8) 
can be written under the polar coordinates as follows:

$$
\psi(r, \theta)=\sum_{n \in \mathbb{Z}} \frac{H_{n}^{(1)}(\kappa r)}{H_{n}^{(1)}(\kappa R)} \hat{\psi}_{n} e^{\mathrm{i} n \theta},
$$

where

$$
\hat{\psi}_{n}=\frac{1}{2 \pi} \int_{0}^{2 \pi} \psi(R, \theta) e^{-\mathrm{i} n \theta} d \theta
$$

and $H_{n}^{(1)}$ is the Hankel function of the first kind with order $n$. To proceed, we introduce the following notation. For any function $u$ defined on the circle $\Gamma_{R}$ having the Fourier expansion:

$$
u=\sum_{n \in \mathbb{Z}} \hat{u}_{n} e^{\mathrm{i} n \theta}, \quad \hat{u}_{n}=\frac{1}{2 \pi} \int_{0}^{2 \pi} u e^{-\mathrm{i} n \theta} d \theta
$$

we define

$$
\begin{aligned}
\|u\|_{H^{1 / 2}\left(\Gamma_{R}\right)}^{2} & =2 \pi \sum_{n \in \mathbb{Z}}\left(1+n^{2}\right)^{1 / 2}\left|\hat{u}_{n}\right|^{2}, \\
\|u\|_{H^{-1 / 2}\left(\Gamma_{R}\right)}^{2} & =2 \pi \sum_{n \in \mathbb{Z}}\left(1+n^{2}\right)^{-1 / 2}\left|\hat{u}_{n}\right|^{2} .
\end{aligned}
$$

Let $\mathcal{T}: H^{1 / 2}\left(\Gamma_{R}\right) \rightarrow H^{-1 / 2}\left(\Gamma_{R}\right)$ be the Dirichlet-to-Neumann operator defined as follows: for any $u \in H^{1 / 2}\left(\Gamma_{R}\right)$,

$$
\mathcal{T} u=\frac{1}{R} \sum h_{n}(\kappa R) \hat{u}_{n} e^{\mathrm{i} n \theta}
$$

where

$$
h_{n}(z)=z \frac{H_{n}^{(1)^{\prime}}(z)}{H_{n}^{(1)}(z)} \quad \text { and } \quad \hat{u}_{n}=\frac{1}{2 \pi} \int_{0}^{2 \pi} u e^{-\mathrm{i} n \theta} d \theta .
$$

The solution written as in (2.1) satisfies

$$
\frac{\partial \psi}{\partial \mathbf{n}}=\mathcal{T} \psi \quad \text { on } \Gamma_{R}
$$

where $\mathbf{n}$ is the unit outward normal to $\Gamma_{R}$.

Following [1], we have

$$
z \frac{H_{n}^{(1)^{\prime}}(z)}{H_{n}^{(1)}(z)}=-\frac{f_{n}(z)}{g_{n}(z)}+i \frac{z}{g_{n}(z)},
$$

where

$$
\begin{aligned}
& f_{n}(z)=c_{n}^{0}+2 c_{n}^{1} \frac{1}{z^{2}}+\cdots+(n+1) c_{n}^{n} \frac{1}{z^{2 n}}, \\
& g_{n}(z)=c_{n}^{0}+c_{n}^{1} \frac{1}{z^{2}}+\cdots+c_{n}^{n} \frac{1}{z^{2 n}},
\end{aligned}
$$

and

$$
c_{n}^{m}=\frac{(m+n) !(2 m) !}{4^{m}(m !)^{2}(n-m) !} .
$$

Evidently, we have

$$
1 \leq-\Re h_{n}(z) \leq n+1 \text { and } 0 \leq \Im h_{n}(z) \leq z
$$


To state the boundary value problem, we introduce the bilinear form $a: H^{1}\left(B_{R}\right) \times H^{1}\left(B_{R}\right) \rightarrow \mathbb{C}$

$$
a(u, v)=(\nabla u, \nabla v)-\kappa^{2}((1+q) u, v)-\langle\mathcal{T} u, v\rangle,
$$

and the linear functional on $H^{1}\left(B_{R}\right)$

$$
b(v)=\kappa^{2}\left(q \phi_{0}, v\right) .
$$

Here we have used the standard inner products

$$
(u, v)=\int_{B_{R}} u \cdot \bar{v} d \mathbf{x} \text { and }\langle u, v\rangle=\int_{\Gamma_{R}} u \cdot \bar{v} d s,
$$

where the overline denotes the complex conjugate. The direct problem (1.7), (1.8) is equivalent to the following weak formulation: to find $\psi \in H^{1}\left(B_{R}\right)$ such that

$$
a(\psi, \xi)=b(\xi), \quad \forall \xi \in H^{1}\left(B_{R}\right)
$$

Throughout the paper, $C$ stands for a positive generic constant whose value may change step by step, but should always be clear from the context.

Lemma 2.1 There exists a constant $C$ such that for any $u \in H^{1 / 2}\left(\Gamma_{R}\right)$ the following inequality holds:

$$
\|\mathcal{T} u\|_{H^{-1 / 2}\left(\Gamma_{R}\right)} \leq C\|u\|_{H^{1 / 2}\left(\Gamma_{R}\right)} .
$$

Furthermore,

$$
-\Re\langle\mathcal{T} u, u\rangle \geq C\|u\|_{L^{2}\left(\Gamma_{R}\right)}^{2} \quad \text { and } \quad \Im\langle\mathcal{T} u, u\rangle \geq 0 .
$$

Proof. For any function $u \in H^{1 / 2}\left(\Gamma_{R}\right)$, we have the Fourier series expansion:

$$
u=\sum_{n \in \mathbb{Z}} \hat{u}_{n} e^{\mathrm{i} n \theta}, \quad \hat{u}_{n}=\frac{1}{2 \pi} \int_{0}^{2 \pi} u e^{-\mathrm{i} n \theta} d \theta .
$$

It follows from the norms for $H^{-1 / 2}\left(\Gamma_{R}\right)$ and $H^{1 / 2}\left(\Gamma_{R}\right)$ and $(2.5)$ that

$$
\|\mathcal{T} u\|_{H^{-1 / 2}\left(\Gamma_{R}\right)}^{2}=\frac{2 \pi}{R^{2}} \sum_{n \in \mathbb{Z}} \frac{\left|h_{n}(\kappa R)\right|^{2}}{\left(1+n^{2}\right)^{1 / 2}}\left|\hat{u}_{n}\right|^{2} \leq C \sum_{n \in \mathbb{Z}}\left(1+n^{2}\right)^{1 / 2}\left|\hat{u}_{n}\right|^{2}=C\|u\|_{H^{1 / 2}\left(\Gamma_{R}\right)}^{2} .
$$

Using the inner product and the Fourier expansion, we arrive at

$$
\langle\mathcal{T} u, u\rangle=\frac{2 \pi}{R} \sum_{n \in \mathbb{Z}} h_{n}(\kappa R)\left|\hat{u}_{n}\right|^{2} .
$$

It follows from (2.5) that

$$
\begin{aligned}
& \Re\langle\mathcal{T} u, u\rangle=\frac{2 \pi}{R} \sum_{n \in \mathbb{Z}} \Re\left(h_{n}(\kappa R)\right)\left|\hat{u}_{n}\right|^{2} \leq-\frac{2 \pi}{R} \sum_{n \in \mathbb{Z}}\left|\hat{u}_{n}\right|^{2}=-C\|u\|_{L^{2}\left(\Gamma_{R}\right)}^{2}, \\
& \Im\langle\mathcal{T} u, u\rangle=\frac{2 \pi}{R} \sum_{n \in \mathbb{Z}} \Im\left(h_{n}(\kappa R)\right)\left|\hat{u}_{n}\right|^{2}>0 .
\end{aligned}
$$


Theorem 2.1 If the wavenumber $\kappa$ is sufficiently small, the variational problem (2.8) admits a unique weak solution in $H^{1}\left(B_{R}\right)$. Further, there is a positive constant $C$ which depends only on $R$, such that

$$
\|\psi\|_{H^{1}\left(B_{R}\right)} \leq C \kappa^{2}\|q\|_{L^{\infty}\left(B_{R}\right)}\left\|\phi_{0}\right\|_{L^{2}\left(B_{R}\right)} .
$$

Proof. Decompose the bilinear form $a$ into $a=a_{1}-\kappa^{2} a_{2}$, where

$$
a_{1}(\psi, \xi)=(\nabla \psi, \nabla \xi)-\langle\mathcal{T} \psi, \xi\rangle \quad \text { and } \quad a_{2}(\psi, \xi)=((1+q) \psi, \xi)
$$

We conclude that $a_{1}$ is coercive from Lemma 2.1

$$
\left|a_{1}(\psi, \psi)\right| \geq C\|\psi\|_{H^{1}\left(B_{R}\right)}^{2},
$$

Next we prove the compactness of $a_{2}$. Define an operator $\mathcal{A}: L^{2}\left(B_{R}\right) \rightarrow H^{1}\left(B_{R}\right)$ by

$$
a_{1}(\mathcal{A} \psi, \xi)=a_{2}(\psi, \xi), \quad \forall \xi \in H^{1}\left(B_{R}\right)
$$

which gives

$$
(\nabla \mathcal{A} \psi, \nabla \xi)-\langle\mathcal{T} \mathcal{A} \psi, \xi\rangle=((1+q) \psi, \xi)
$$

Using the Lax-Milgram lemma and Lemma 2.1, we obtain

$$
\|\mathcal{A} \psi\|_{H^{1}\left(B_{R}\right)} \leq C\|\psi\|_{L^{2}\left(B_{R}\right)} .
$$

Thus, $\mathcal{A}$ is bounded from $L^{2}\left(B_{R}\right)$ to $H^{1}\left(B_{R}\right)$ and $H^{1}\left(B_{R}\right)$ is compactly embedded into $L^{2}\left(B_{R}\right)$. Hence, $\mathcal{A}$ is a compact operator.

Define a function $u \in L^{2}\left(B_{R}\right)$ by requiring $u \in H^{1}\left(B_{R}\right)$ and satisfying

$$
a_{1}(u, \xi)=b(\xi), \quad \forall \xi \in H^{1}\left(B_{R}\right) .
$$

It follows from the Lax-Milgram lemma again that

$$
\|u\|_{H^{1}\left(B_{R}\right)} \leq C \kappa^{2}\|q\|_{L^{\infty}\left(B_{R}\right)}\left\|\phi_{0}\right\|_{L^{2}\left(B_{R}\right)} .
$$

Using the operator $\mathcal{A}$, we can see that the problem (2.8) is equivalent to find $\psi \in L^{2}\left(B_{R}\right)$ such that

$$
\left(\mathcal{I}-\kappa^{2} \mathcal{A}\right) \psi=u
$$

When the wavenumber $\kappa$ is small enough, the operator $\mathcal{I}-\kappa^{2} \mathcal{A}$ has a uniformly bounded inverse. We then have the estimate

$$
\|\psi\|_{L^{2}\left(B_{R}\right)} \leq C\|u\|_{L^{2}\left(B_{R}\right)},
$$

where the constant $C$ is independent of $\kappa$. Rearranging (2.11), we have $\psi=u-\kappa^{2} \mathcal{A} \psi$, so $\psi \in H^{1}\left(B_{R}\right)$ and, by the estimate (2.10) for the operator $\mathcal{A}$, we have

$$
\|\psi\|_{H^{1}\left(B_{R}\right)} \leq\|u\|_{H^{1}\left(B_{R}\right)}+C \kappa^{2}\|\psi\|_{L^{2}\left(B_{R}\right)} .
$$

The proof is complete by combining the above estimate and (2.11). 
Remark 2.1 For the propagating plane wave, the estimated (2.9) can be written as

$$
\|\psi\|_{H^{1}\left(B_{R}\right)} \leq C \kappa^{2}|\Omega|^{1 / 2}\|q\|_{L^{\infty}\left(B_{R}\right)} .
$$

The energy estimate of the scattered field (2.14) provide a criterion for weak scattering. From this estimate, it is easily seen that, fixing any two of the three quantities, i.e., the wavenumber, the compact support of the scatterer $\Omega$, and the $L^{\infty}\left(B_{R}\right)$ norm of the scatterer, the scattering is weak when the third one is small. Especially for the given scatterer q, i.e., the norm and the compact support are fixed, the scattering is weak when the wavenumber is small.

Remark 2.2 For a general wavenumber, from (2.12) the uniqueness and existence follow from the Fredholm alternative, i.e., if $\kappa$ is not the eigenvalue for the Helmholtz equation in the domain $B_{R}$, then the operator $\mathcal{I}-\kappa^{2} \mathcal{A}$ has a bounded inverse. However, the bound depends on the wavenumber. Therefore, the constant $C$ in the estimate (2.9) depends on the wavenumber.

Theorem 2.2 Given the scatterer $q \in L^{\infty}\left(B_{R}\right)$, for all but possibly a discrete set of wavenumbers, the variational problem (2.8) admits a unique weak solution in $H^{1}\left(B_{R}\right)$. Further, there is a positive constant $C$ which depends on $R$ and $\kappa$, such that

$$
\|\psi\|_{H^{1}\left(B_{R}\right)} \leq C\|q\|_{L^{\infty}\left(B_{R}\right)}\left\|\phi_{0}\right\|_{L^{2}\left(B_{R}\right)} .
$$

Remark 2.3 For the evanescent plane wave with $|\eta|>\kappa$, the estimate (2.14) can be written as

$$
\|\psi\|_{H^{1}\left(B_{R}\right)} \leq C\left(\eta^{2}-\kappa^{2}\right)^{-1 / 4}\|q\|_{L^{\infty}\left(B_{R}\right)},
$$

where the constant $C$ depends on $\kappa$ and $R$. The above energy estimate also provides a criterion for the weak scattering. For a fixed wavenumber $\kappa$ and a scatterer $q$, the scattered field is weak if the spatial frequency of the incident wave $|\eta|$ is large.

\section{$3 \quad$ Initial guess}

In this section, we discuss how to generate an initial guess for the proposed recursive linearization method based on either the linearized Lippmann-Schwinger integral equation when the weak scattering is valid, or the multiple signal classification algorithm when the weak scattering may not be valid.

\subsection{Born approximation}

Rewrite (1.7) as

$$
\Delta \psi+\kappa^{2} \psi=-\kappa^{2} q\left(\phi_{0}+\psi\right)
$$


From the energy estimates (2.14) and (2.16), the scattered field is weak when the wavenumber $\kappa$ is small or when the spatial frequency $|\eta|$ is large. By dropping the scattered field at the right hand side of (3.1) under the weak scattering, we obtain

$$
\Delta \psi+\kappa^{2} \psi=-\kappa^{2} q \phi_{0}
$$

which is the well-known Born approximation.

Consider an auxiliary function $\psi_{0}(\mathbf{x})=e^{\mathrm{i} \kappa \mathbf{p} \cdot \mathbf{x}}, \mathbf{p}=(\cos \beta, \sin \beta), \beta \in[0,2 \pi]$. This auxiliary function represents propagating plane waves and hence satisfies (1.5). Multiplying (3.2) by $\psi_{0}$ and integrating over $B_{R}$ on both sides, we have

$$
\int_{B_{R}} \psi_{0} \Delta \psi d \mathbf{x}+\kappa^{2} \int_{B_{R}} \psi_{0} \psi d \mathbf{x}=-\kappa^{2} \int_{B_{R}} q \phi_{0} \psi_{0} d \mathbf{x}
$$

Integration by parts yields

$$
\int_{B_{R}} \psi \Delta \psi_{0} d \mathbf{x}+\int_{\Gamma_{R}}\left(\psi_{0} \frac{\partial \psi}{\partial \mathbf{n}}-\psi \frac{\partial \psi_{0}}{\partial \mathbf{n}}\right) d s+\kappa^{2} \int_{B_{R}} \psi_{0} \psi d \mathbf{x}=-\kappa^{2} \int_{B_{R}} q \phi_{0} \psi_{0} d \mathbf{x}
$$

We have by noting (1.5) and the boundary condition (2.3) that

$$
\int_{B_{R}} q \phi_{0} \psi_{0} d \mathbf{x}=\frac{1}{\kappa^{2}} \int_{\Gamma_{R}}\left(\psi \frac{\partial \psi_{0}}{\partial \mathbf{n}}-\psi_{0} \mathcal{T} \psi\right) d s
$$

Using the special form of the incident wave and the auxiliary function, we then get

$$
\int_{B_{R}} q e^{\mathrm{i}(\mathbf{k}+\kappa \mathbf{p}) \cdot \mathbf{x}} d \mathbf{x}=\frac{1}{\kappa^{2}} \int_{\Gamma_{R}}\left(\psi \frac{\partial \psi_{0}}{\partial \mathbf{n}}-\psi_{0} \mathcal{T} \psi\right) d s .
$$

When the incident waves are propagating waves, i.e., $\mathbf{k}=\kappa \mathbf{d}$, the linear integral equation (3.6) becomes

$$
\int_{B_{R}} q e^{\mathrm{i} \kappa(\mathbf{d}+\mathbf{p}) \cdot \mathbf{x}} d \mathbf{x}=\frac{1}{\kappa^{2}} \int_{\Gamma_{R}}\left(\psi \frac{\partial \psi_{0}}{\partial \mathbf{n}}-\psi_{0} \mathcal{T} \psi\right) d s .
$$

Since the scatterer $q$ has a compact support, we use the notation

$$
\hat{q}(\xi)=\int_{B_{R}} q(\mathbf{x}) e^{\mathrm{i} \kappa(\mathbf{p}+\mathbf{n}) \cdot \mathbf{x}},
$$

where $\hat{q}(\xi)$ is the Fourier transform of $q(\mathbf{x})$ with $\xi=\kappa(\mathbf{p}+\mathbf{d})$. It is obvious that the domain $[0,2 \pi] \times[0,2 \pi]$ of $(\alpha, \beta)$ corresponds to the ball $\left\{\xi \in \mathbb{R}^{2}:|\xi| \leq 2 \kappa\right\}$. Thus, the Fourier modes of $q(\mathbf{x})$ in the ball $\{\xi:|\xi| \leq 2 \kappa\}$ can be determined. The scattering data with higher wavenumber must be used in order to recover more modes of the true scatterer.

Define the data

$$
D(\xi)= \begin{cases}\frac{1}{\kappa^{2}} \int_{\Gamma_{R}}\left(\psi \frac{\partial \psi_{0}}{\partial \mathbf{n}}-\psi_{0} \mathcal{T} \psi\right) d s & \text { for }|\xi| \leq 2 \kappa, \\ 0 & \text { for }|\xi|>2 \kappa .\end{cases}
$$

The equation (3.7) can be formally reformulated as

$$
\hat{q}(\xi)=D(\xi)
$$


Taking the inverse Fourier transform of (3.8) leads to an initial approximation

$$
q(\mathbf{x})=\frac{1}{(2 \pi)^{2}} \int_{\mathbb{R}^{2}} e^{-\mathrm{ix} \cdot \xi} D(\xi) d \xi
$$

which may be implemented by using the fast Fourier transform.

When the incident waves are evanescent, i.e., $\mathbf{k}=\left(\eta, \mathrm{i} \sqrt{\eta^{2}-\kappa^{2}}\right)$, the linear integral equation (3.6) becomes

$$
\int_{B_{R}} q(\mathbf{x}) e^{\mathrm{i}(\kappa \cos \beta+\eta) x_{1}} e^{\left(\mathrm{i} \kappa \sin \beta-\sqrt{\eta^{2}-\kappa^{2}}\right) x_{2}} d \mathbf{x}=\frac{1}{\kappa^{2}} \int_{\Gamma_{R}}\left(\psi \frac{\partial \psi_{0}}{\partial \mathbf{n}}-\psi_{0} \mathcal{T} \psi\right) d s .
$$

Since the scatterer $q(\mathbf{x})$ has a compact support, (3.10) can be rewritten as

$$
\int_{-\infty}^{\infty} \hat{q}\left(\xi, x_{2}\right) e^{\left(\mathrm{i} \kappa \sin \beta-\sqrt{\eta^{2}-\kappa^{2}}\right) x_{2}} d x_{2}=D(\xi, \eta),
$$

where $\xi=\kappa \cos \beta+\eta$ and $\hat{q}\left(\xi, x_{2}\right)$ is the Fourier transform of $q(\mathbf{x})$ with respect to $x_{1}$. When the spatial frequency $|\eta|$ is large, the incident wave penetrates a thin layer of the scatterer. Thus, the Born approximation allows a reconstruction containing information of the true scatterer in that thin layer. For propagating plane incident waves, the inversion involves data related to the scatterer through the Fourier transform in the case of weak scattering. For evanescent plane wave, the inversion involves data related to the scatterer through a Fourier (with respect to $x_{1}$ )-Laplace (with respect to $x_{2}$ ) transform in the case of the weak scattering.

Introduce the integral kernel

$$
\mathcal{K}\left(\xi, \eta ; x_{2}\right)=e^{\left(\mathrm{i} \kappa \sin \beta-\sqrt{\eta^{2}-\kappa^{2}}\right) x_{2}} .
$$

The integral equation (3.11) can be formally written as

$$
\mathcal{K}(\xi) \hat{q}(\xi)=D(\xi)
$$

In practice, Equation (3.12) is implemented by using the method of least squares with Tikhonov regularization

$$
\hat{q}(\xi)=\left(\lambda \mathcal{I}+\mathcal{K}^{*} \mathcal{K}\right)^{-1} \mathcal{K}^{*} D(\xi),
$$

where $\lambda$ is a small positive number, $\mathcal{I}$ is the identity operator, and $\mathcal{K}^{*}$ is the adjoint operator of $\mathcal{K}$. Once $\hat{q}\left(\xi, x_{2}\right)$ is available, an approximation of $q(\mathbf{x})$ may be obtained from the inverse Fourier transform.

\subsection{MUSIC algorithm}

The MUSIC (MUltiple SIgnal Classification) algorithm for extended scatterers proposed in [27] is used to generate an image for the shape of the scatterer. The MUSIC algorithm for point scatterers may be found in [25]. The image may be further converted into a level set representation for the scatterer through image processing. See also [2] for an up-to-date discussion on various types of mathematical imaging methods. 


\subsubsection{The MUSIC algorithm for extended scatterers}

Consider plane incident waves illuminating from $m$ evenly spaced angles with a certain wavenumber. The scattered fields are recorded on $\partial \Omega$ with the same $m$ evenly spaced angles. The data collected forms an $m$-by- $m$ matrix, denoted by $P$, which is known as the response matrix. For simplicity of discussion, here we have the incident plane wave directions coincide with the recorded scattered field directions. However, the MUSIC algorithm and our continuation method to be discussed later can both handle the general case where the number of incident plane wave directions is different from the number of recorded scattered field directions and the directions do not coincide.

Let $P=U \Sigma V^{H}$ be the singular value decomposition of the response matrix. Define the illumination vector

$$
\mathbf{g}(\mathbf{x})=\left[e^{\mathrm{i} k \mathbf{x} \cdot \mathbf{d}_{1}}, \ldots, e^{\mathrm{i} k \mathbf{x} \cdot \mathbf{d}_{m}}\right]^{T},
$$

where $\mathbf{d}_{j}$ are the propagation directions of incident waves and $\mathbf{x}$ is any point in the space. The MUSIC imaging function may be introduced:

$$
I(\mathbf{x})=\frac{1}{\|\mathbf{g}(\mathbf{x})\|_{2}^{2}-\sum_{\ell=1}^{s}\left|\mathbf{g}(\mathbf{x})^{H} \mathbf{u}_{\ell}\right|^{2}},
$$

where $\mathbf{u}_{\ell}$ is the $\ell$ th column of the matrix $U$ and the number of singular vectors $s$ that spans the signal space is determined by the resolution analysis based thresholding algorithm in [27].

The imaging function (3.14) provides an image for the boundary of the scatterer, which may be further converted into a level set representation for the scatterer.

\subsubsection{Image processing and the level set function}

In this section, we briefly describe an image processing to convert the image for the boundary of the scatterer into a level set representation, which leads to an initial guess. Additional discussions and results are available in [4].

There are many edge detector algorithms in the literature $[12,13,31]$. Here, we employ a relatively simple approach. Starting with a large domain enclosing the scatterer, we minimize the cost functional

$$
C(\partial \Omega)=\int_{\partial \Omega} f(\mathbf{x}) d s
$$

where $f(\mathbf{x})=1$ if the imaging function $I(\mathbf{x})$ is larger than some threshold and $f(\mathbf{x})=100$ otherwise. In other words, on the boundary of the scatterer, $f$ is small. It makes the curve shrink to the boundary of the scatterer by minimizing the functional (3.15). In fact, the function $f$ acts as the weight for the curvature-based force in the curve evolution.

Let $\varphi(\mathbf{x})$ be a level set function that characterizes the curve $\partial \Omega$, i.e., $\varphi(\mathbf{x})=0$ on $\partial \Omega, \varphi(\mathbf{x})>0$ outside $\Omega ; \varphi(\mathbf{x})<0$ inside $\Omega$. The cost functional can be formulated as [40]

$$
C(\partial \Omega)=W(\varphi)=\int_{\mathbb{R}^{2}} f(\mathbf{x}) \delta(\varphi)|\nabla \varphi| d \mathbf{x},
$$

where $\delta$ is the Dirac delta function. Taking the derivative with respect to the evolution time $t$, we have

$$
\frac{d W}{d t}=\int_{\mathbb{R}^{2}}\left(\delta^{\prime}(\varphi)|\nabla \varphi| \varphi_{t}+\delta(\varphi) \frac{\nabla \varphi}{|\nabla \varphi|} \cdot \nabla\left(\varphi_{t}\right)\right) f(\mathbf{x}) d \mathbf{x}
$$


The level set formulation for shape evolution with the normal velocity $v(\mathbf{x})$ is [36]

$$
\varphi_{t}=-v(\mathbf{x})|\nabla \varphi|
$$

By substituting (3.18) into (3.17) and using $\delta^{\prime}(\varphi) \nabla \varphi=\nabla(\delta(\varphi))$, we obtain

$$
\frac{d W}{d t}=-\int_{\mathbb{R}^{2}}\left(v(\mathbf{x}) \nabla(\delta(\varphi)) \cdot \nabla \varphi+\delta(\varphi) \frac{\nabla \varphi}{|\nabla \varphi|} \cdot \nabla(v(\mathbf{x})|\nabla \varphi|)\right) f(\mathbf{x}) d \mathbf{x} .
$$

Although the evolution velocity is only defined on the moving curve initially, it can be extended by a constant normal extension away from the curve. Since $\nabla \varphi$ is in the normal direction, we have $\nabla v \cdot \nabla \varphi=0$. Therefore, the equation (3.19) can be rewritten as

$$
\frac{d W}{d t}=-\int_{\mathbb{R}^{2}}\left(\nabla(\delta(\varphi)) \cdot \nabla \varphi+\delta(\varphi) \frac{\nabla \varphi}{|\nabla \varphi|} \cdot \nabla(|\nabla \varphi|)\right) v(\mathbf{x}) f(\mathbf{x}) d \mathbf{x} .
$$

It follows from the divergence theorem on the first term of the right hand side of (3.20) that

$$
\frac{d W}{d t}=\int_{\mathbb{R}^{2}} \delta(\varphi)\left(\nabla \cdot(v(\mathbf{x}) f(\mathbf{x}) \nabla \varphi)-\frac{\nabla \varphi}{|\nabla \varphi|} \cdot \nabla(|\nabla \varphi|) v(\mathbf{x}) f(\mathbf{x})\right) d \mathbf{x} .
$$

Simple calculations from the product rule yield

$$
\frac{d W}{d t}=\int_{\mathbb{R}^{2}} \delta(\varphi)|\nabla \varphi| v(\mathbf{x}) \nabla \cdot\left(f(\mathbf{x}) \frac{\nabla \varphi}{|\nabla \varphi|}\right) d \mathbf{x}
$$

which can be written as a curve integral

$$
\frac{d W}{d t}=\int_{\partial \Omega} v(\mathbf{x}) \nabla \cdot\left(f(\mathbf{x}) \frac{\nabla \varphi}{|\nabla \varphi|}\right) d s
$$

Let $v(\mathbf{x})=-\nabla \cdot\left(f(\mathbf{x}) \frac{\nabla \varphi}{|\nabla \varphi|}\right)$. By substituting it into (3.18), we arrive at the gradient flow for the level set function

$$
\varphi_{t}=|\nabla \varphi| \nabla \cdot\left(f(\mathbf{x}) \frac{\nabla \varphi}{|\nabla \varphi|}\right) .
$$

By using such a normal velocity, we always have $d W / d t<0$, i.e., the cost functional decreases monotonically in the shape evolution. In practice, a local level set method [37] with reinitialization using a time marching scheme [38] is employed for solving (3.24).

Starting with a box containing all scatterers, the evolution will stop at the convex envelope of the shapes for scatterers in the MUSIC imaging result. The level set function representing the shape of the envelope may be selected as an initial guess.

\section{Recursive linearization}

In this section, two regularized recursive linearization methods for solving the inverse medium scattering problem with multiple frequency and single frequency are proposed, respectively.

One of the recursive linearization methods, obtained by continuation on the wavenumber $\kappa$, requires multiple frequency scattering data. At each wavenumber $\kappa$, the algorithm determines a forward 
model which produces the prescribed scattering data. At low wavenumber $\kappa$, the scattered field is weak. Consequently, the nonlinear equation become essentially a linear one. The algorithm first solves this nearly linear equation at the lowest $\kappa$ to obtain low-frequency modes of the true scatterer. The approximation is then used to linearize the nonlinear equation at the next higher $\kappa$ to produce a better approximation which contains more modes of the true scatterer. This process is continued until a sufficiently high wavenumber $\kappa$ where the dominant modes of the scatterer are essentially recovered.

Another recursive linearization method, obtained by continuation method on the spatial frequency of a one-parameter family of incident plane waves, requires only single frequency scattering data. At each transverse part of the incident wave, the algorithm determines a forward model which produces the prescribed scattering data. Since the incident wave at a high spatial frequency can only penetrate a thin layer of the scatterer, the scattered field is weak. Consequently, the nonlinear equation becomes essentially linear, known as the Born approximation. The algorithm first solves this nearly linear equation at the largest $|\eta|$ to obtain an approximation of the scatterer. This approximation is then used to linearize the nonlinear equation at the next smaller spatial frequency of the incident wave, which can penetrate a thicker layer of the scatterer, to produce a better approximation. When the spatial frequency, $|\eta|$, is smaller than the fixed wavenumber $\kappa$, the incident wave becomes usual propagating plane wave, and the whole scatterer is illuminated. This process is continued until the spatial frequency is zero, where the approximation of the scatterer is considered as the final reconstruction.

\subsubsection{Multiple frequency}

As discussed in the previous section, when the wavenumber $\kappa$ is small, the Born approximation allows a reconstruction of those low Fourier modes for the function $q$. We now describe a procedure that recursively determines better approximations $q_{\kappa}$ at $\kappa=\kappa_{l}$ for $l=1,2, \ldots$ with the increasing wavenumbers. Suppose now that an approximation of the scatterer, $q_{\tilde{\kappa}}$, has been recovered at some wavenumber $\tilde{\kappa}$, and that the wavenumber $\kappa$ is slightly larger that $\tilde{\kappa}$. We wish to determine $q_{\kappa}$, or equivalently, to determine the perturbation

$$
\delta q=q_{\kappa}-q_{\tilde{\kappa}}
$$

For the reconstructed scatterer $q_{\tilde{\kappa}}$, we solve at the wavenumber $\kappa$ the forward scattering problem

$$
\begin{aligned}
\Delta \tilde{\psi}_{i}+\kappa^{2}\left(1+q_{\tilde{\kappa}}\right) \tilde{\psi}_{i} & =-\kappa^{2} q_{\tilde{\kappa}} \phi_{0}^{(i)} \quad \text { in } B_{R}, \\
\frac{\partial \tilde{\psi}_{i}}{\partial \mathbf{n}} & =\mathcal{T} \tilde{\psi}_{i} \quad \text { on } \Gamma_{R},
\end{aligned}
$$

where $\phi_{0}^{(i)}$ is the incident with incident angle $\alpha_{i}, i=1, \ldots, n$.

For the scatterer $q_{\kappa}$, we have

$$
\begin{aligned}
\Delta \psi_{i}+\kappa^{2}\left(1+q_{\kappa}\right) \psi_{i} & =-\kappa^{2} q_{\kappa} \phi_{0}^{(i)} \quad \text { in } B_{R}, \\
\frac{\partial \psi_{i}}{\partial \mathbf{n}} & =\mathcal{T} \psi_{i} \quad \text { on } \Gamma_{R} .
\end{aligned}
$$


Subtracting (4.1) from (4.3) and omitting the second order smallness in $\delta q$ and in $\delta \psi_{i}=\psi_{i}-\tilde{\psi}_{i}$, we obtain

$$
\begin{aligned}
\Delta \delta \psi_{i}+\kappa^{2}\left(1+q_{\tilde{\kappa}}\right) \delta u_{i}^{\mathrm{s}} & =-\kappa^{2} \delta q\left(\phi_{0}^{(i)}+\tilde{\psi}_{i}\right) \quad \text { in } B_{R}, \\
\frac{\partial \delta \psi_{i}}{\partial \mathbf{n}} & =\mathcal{T} \delta \psi_{i} \quad \text { on } \Gamma_{R} .
\end{aligned}
$$

Given a solution $\psi_{i}$ of (4.3), we define the measurements

$$
M \psi_{i}(\mathbf{x})=\left[\psi_{i}\left(\mathbf{x}_{1}\right), \ldots, \psi_{i}\left(\mathbf{x}_{m}\right)\right]^{T} .
$$

The measurement operator $M$ is well defined and maps the scattered field to a vector of complex numbers in $\mathbb{C}^{m}$, which consists of point measurements of the scattered field at $\mathbf{x}_{j}, j=1, \ldots, m$.

For the scatterer $q_{\kappa}$ and the transmitted field $\phi_{0}^{(i)}$, we define the forward scattering operator

$$
S\left(q_{\kappa}, \phi_{0}^{(i)}\right)=M \psi_{i}
$$

It is easily seen that the forward scattering operator $S\left(q_{\kappa}, \phi_{0}^{(i)}\right)$ is linear with respect to $\phi_{0}^{(i)}$ but nonlinear with respect to $q_{\kappa}$. For simplicity, we denote $S\left(q_{\kappa}, \phi_{0}^{(i)}\right)$ by $S_{i}\left(q_{\kappa}\right)$. Let $S_{i}^{\prime}\left(q_{\tilde{\kappa}}\right)$ be the Fréchet derivative of $S_{i}\left(q_{\kappa}\right)$ and denote the residual operator

$$
R_{i}\left(q_{\tilde{\kappa}}\right)=M\left(\delta \psi_{i}\right)
$$

It follows from the linearization of the nonlinear equation (4.8) that

$$
S_{i}^{\prime}\left(q_{\tilde{\kappa}}\right) \delta q=R_{i}\left(q_{\tilde{\kappa}}\right) .
$$

Applying the Landweber iteration [22] to the linearized equation (4.10) yields

$$
\delta q=\tau S_{i}^{\prime}\left(q_{\tilde{\kappa}}\right)^{*} R_{i}\left(q_{\tilde{\kappa}}\right),
$$

where $\tau$ is a positive relaxation parameter and $S_{i}^{\prime}\left(q_{\tilde{\kappa}}\right)^{*}$ is the adjoint operator of $S_{i}^{\prime}\left(q_{\tilde{\kappa}}\right)$.

In order to compute the correction $\delta q$, we need some efficient way to compute $S_{i}^{\prime}\left(q_{\tilde{\kappa}}\right)^{*} R_{i}\left(q_{\tilde{\kappa}}\right)$. Let $R_{i}\left(q_{\tilde{\kappa}}\right)=\left[\zeta_{i 1}, \ldots, \zeta_{i m}\right]^{T} \in \mathbb{C}^{m}$. Consider the adjoint problem

$$
\begin{aligned}
\Delta w_{i}+\kappa^{2}\left(1+q_{\tilde{\kappa}}\right) w_{i} & =-\kappa^{2} \sum_{j=1}^{m} \zeta_{i j} \delta\left(\mathbf{x}-\mathbf{x}_{j}\right) \quad \text { in } B_{R}, \\
\frac{\partial w_{i}}{\partial \mathbf{n}} & =\mathcal{T}^{*} w_{i} \quad \text { on } \Gamma_{R}
\end{aligned}
$$

where the operator $\mathcal{T}^{*}$ is defined as

$$
\mathcal{T}^{*} u=\kappa \sum_{n \in \mathbb{Z}} \overline{\left(\frac{H_{n}^{(1)^{\prime}}(\kappa R)}{H_{n}^{(1)}(\kappa R)}\right)} \hat{u}_{n} e^{\mathrm{i} n \theta}, \quad \hat{u}_{n}=\frac{1}{2 \pi} \int_{0}^{2 \pi} u e^{-\mathrm{i} n \theta} d \theta .
$$

Multiplying (4.5) with the complex conjugate of $w_{i}$ and integrating over $B_{R}$ on both sides, we obtain

$$
\int_{B_{R}} \Delta \delta \psi_{i} \bar{w}_{i} d \mathbf{x}+\int_{B_{R}} \kappa^{2}\left(1+q_{\tilde{\kappa}}\right) \delta \psi_{i} \bar{w}_{i} d \mathbf{x}=-\kappa^{2} \int_{B_{R}} \delta q\left(\phi_{0}^{(i)}+\tilde{\psi}_{i}\right) \bar{w}_{i} d \mathbf{x} .
$$


Using Green's formula, we have

$$
\begin{aligned}
\int_{B_{R}}\left(\Delta \bar{w}_{i}+\kappa^{2}\left(1+q_{\tilde{\kappa}}\right) \bar{w}_{i}\right) \delta \psi_{i} d \mathbf{x} & +\int_{\Gamma_{R}}\left(\frac{\partial \bar{w}_{i}}{\partial \mathbf{n}} \delta \psi_{i}-\frac{\partial \delta \psi_{i}}{\partial \mathbf{n}} \bar{w}_{i}\right) d s \\
& =-\kappa^{2} \int_{B_{R}} \delta q\left(\phi_{0}^{(i)}+\tilde{\psi}_{i}\right) \bar{\psi}_{i} d \mathbf{x} .
\end{aligned}
$$

It follows from the adjoint equation (4.12) that

$$
\sum_{j=1}^{m} \delta \psi_{i}\left(\mathbf{x}_{j}\right) \bar{\zeta}_{i j}=\int_{B_{R}} \delta q\left(\phi_{0}^{(i)}+\tilde{\psi}_{i}\right) \bar{w}_{i} d \mathbf{x} .
$$

Noting (4.9), (4.10), and the adjoint operator $S_{i}^{\prime}\left(q_{\tilde{\kappa}}\right)^{*}$, the left-hand side of (4.14) may be deduced

$$
\begin{aligned}
\sum_{j=1}^{m} \delta u_{i}^{\mathrm{s}}\left(\mathbf{x}_{j}\right) \bar{\zeta}_{i j} & =\left\langle M\left(\delta u_{i}^{\mathrm{s}}\right), R_{i}\left(q_{\tilde{\kappa}}\right)\right\rangle_{\mathbb{C}^{m}}=\left\langle S_{i}^{\prime}\left(q_{\tilde{\kappa}}\right) \delta q, R_{i}\left(q_{\tilde{\kappa}}\right)\right\rangle_{\mathbb{C}^{m}} \\
& =\left\langle\delta q, S_{i}^{\prime}\left(q_{\tilde{\kappa}}\right)^{*} R_{i}\left(q_{\tilde{\kappa}}\right)\right\rangle_{L^{2}\left(B_{R}\right)}=\int_{B_{R}} \delta q \overline{S^{\prime}\left(q_{\tilde{k}}\right)^{*} R_{i}\left(q_{\tilde{\kappa}}\right)} d \mathbf{x} .
\end{aligned}
$$

where $\langle\cdot, \cdot\rangle_{\mathbb{C}^{m}}$ and $\langle\cdot, \cdot\rangle_{L^{2}\left(B_{R}\right)}$ are the standard inner-products defined in the complex vector space $\mathbb{C}^{m}$ and the square integrable functional space $L^{2}\left(B_{R}\right)$.

Combining (4.14) and (4.15) yields

$$
\int_{B_{R}} \delta q \overline{S^{\prime}\left(q_{\tilde{\kappa}}\right)^{*} R_{i}\left(q_{\tilde{\kappa}}\right)} d \mathbf{x}=\int_{B_{R}} \delta q\left(\phi_{0}^{(i)}+\tilde{\psi}_{i}\right) \bar{w}_{i} d \mathbf{x}
$$

which holds for any $\delta q$. It follows that

$$
S^{\prime}\left(q_{\tilde{\kappa}}\right)^{*} R_{i}\left(q_{\tilde{\kappa}}\right)=\left(\overline{\phi_{0}^{(i)}}+\overline{\tilde{\psi}_{i}}\right) w_{i}
$$

Using the above result, Equation (4.11) can be written as

$$
\delta q=\tau\left(\overline{\phi_{0}^{(i)}}+\overline{\tilde{\psi}_{i}}\right) w_{i}
$$

Thus, for each incident wave, we solve one forward problem (4.1), (4.2) and one adjoint problem (4.12), (4.13). Once $\delta q$ is determined, $q_{\kappa}$ is updated by $q_{\tilde{\kappa}}+\delta q$. After completing the $p$ th sweep, we get the reconstructed scatterer $q_{\kappa}$ at the wavenumber $\kappa$.

\subsubsection{Single frequency}

As discussed in the previous section, when the spatial frequency $|\eta|$ is large, the Born approximation allows a reconstruction of the thin layer for the true scatterer. In this section, a regularized recursive linearization method for solving the two-dimensional Helmholtz equation at fixed frequency is proposed.

Choose a large positive number $\eta_{\max }$ and divide the interval $\left[0, \eta_{\max }\right]$ into $N$ subdivisions with the endpoints $\left\{\eta_{0}, \eta_{1}, \ldots, \eta_{N}\right\}$, where $\eta_{0}=0, \eta_{N}=\eta_{\max }$, and $\eta_{n-1}<\eta_{n}$ for $1 \leq n \leq N$. We intend to obtain $q_{\eta}$ recursively at $\eta=\eta_{N}, \eta_{N-1}, \ldots, \eta_{0}$. 
Suppose now that the scatterer $q_{\tilde{\eta}}$ has been recovered at some $\tilde{\eta}=\eta_{n+1}$ and that $\eta=\eta_{n}$ is slightly less than $\tilde{\eta}$. We wish to determine $q_{\eta}$, or equivalently, to determine the perturbation

$$
\delta q=q_{\eta}-q_{\tilde{\eta}}
$$

For the reconstructed scatterer $q_{\tilde{\eta}}$, we solve at the spatial frequency $\eta$ the forward scattering problem

$$
\begin{aligned}
\Delta \tilde{\psi}_{i}+\kappa^{2}\left(1+q_{\tilde{\eta}}\right) \tilde{\psi}_{i} & =-\kappa^{2} q_{\tilde{\eta}} \phi_{0}^{(i)} \quad \text { in } B_{R} \\
\frac{\partial \tilde{\psi}_{i}}{\partial \mathbf{n}} & =\mathcal{T} \tilde{\psi}_{i} \quad \text { on } \Gamma_{R}
\end{aligned}
$$

where the incident wave $\phi_{0}^{(i)}=e^{\mathrm{i} \eta_{i} x_{1}+\mathrm{i} k\left(\eta_{i}\right) x_{2}},\left|\eta_{i}\right| \geq \eta$.

For the scatterer $q_{\eta}$, we have

$$
\begin{aligned}
\Delta \psi_{i}+\kappa\left(1+q_{\eta}\right) \psi_{i} & =-\kappa^{2} q_{\eta} \phi_{0}^{(i)} \quad \text { in } B_{R}, \\
\frac{\partial \psi_{i}}{\partial \mathbf{n}} & =\mathcal{T} \psi_{i} \quad \text { on } \Gamma_{R} .
\end{aligned}
$$

Subtracting (4.18), (4.19) from (4.20), (4.21) and omitting the second-order smallness in $\delta q$ and in $\delta \psi_{i}=\psi_{i}-\tilde{\psi}_{i}$, we obtain

$$
\begin{aligned}
\Delta \delta \psi_{i}+\kappa^{2}\left(1+q_{\tilde{\eta}}\right) \delta \psi_{i} & =-\kappa^{2} \delta q\left(\phi_{0}^{(i)}+\tilde{\psi}_{i}\right) \quad \text { in } B_{R}, \\
\frac{\partial \delta \psi_{i}}{\partial \mathbf{n}} & =\mathcal{T} \delta \psi_{i} \quad \text { on } \Gamma_{R} .
\end{aligned}
$$

In order to compute the update $\delta q$, we may similarly consider the adjoint equation (4.12) and (4.13). Following from the same procedure as that in the case of multiple frequency, we may have again (4.17).

So for each incident wave with a transverse part $\eta_{j}$, we have to solve one forward problem (4.1), (4.2) along with one adjoint problem (4.12), (4.13). Since the adjoint problem has a similar variational form as the forward problem. Essentially, we need to compute two forward problems at each sweep. Once $\delta q$ is determined, $q_{\tilde{\eta}}$ is updated by $q_{\tilde{\eta}}+\delta q$. After completing sweeps with $\left|\eta_{j}\right| \geq \eta$, we get the reconstructed scatterer $q_{\eta}$ at the spatial frequency $\eta$.

\section{$5 \quad$ Numerical experiments}

In order to illustrate the performance of our algorithms, we present three numerical examples. The scattering data are obtained by numerical solution of the direct scattering problem, which is implemented by using the finite element method with a perfectly matched layer technique. For stability analysis, some relative random noise is added to the data, i.e., the scattered field takes the form

$$
\psi\left(\mathbf{x}_{j}\right):=(1+\sigma \text { rand }) \psi\left(\mathbf{x}_{j}\right), j=0, \ldots, m .
$$

Here, rand gives uniformly distributed random numbers in $[-1,1]$ and $\sigma$ is a noise level parameter taken to be 0.05 in our numerical experiments. Define the relative error by

$$
e_{2}=\frac{\left(\sum_{i, j}\left|q_{i j}-\tilde{q}_{i j}\right|^{2}\right)^{1 / 2}}{\left(\sum_{i, j}\left|q_{i j}\right|^{2}\right)^{1 / 2}},
$$




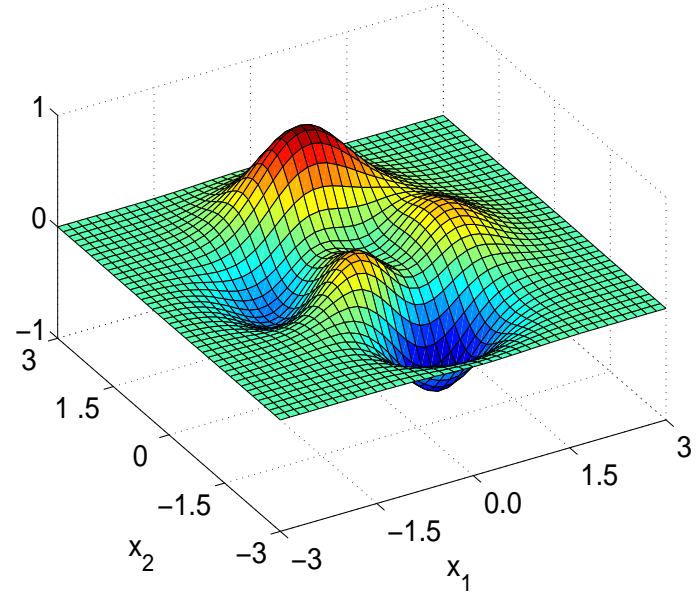

(a)

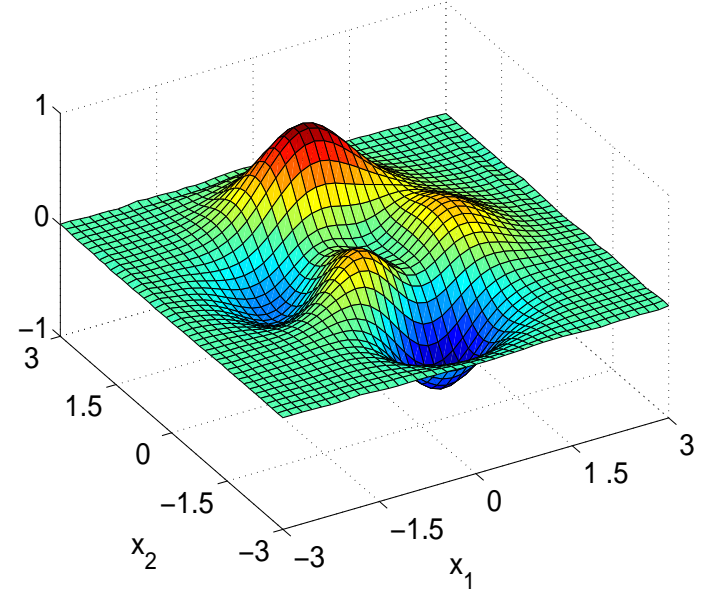

(b)

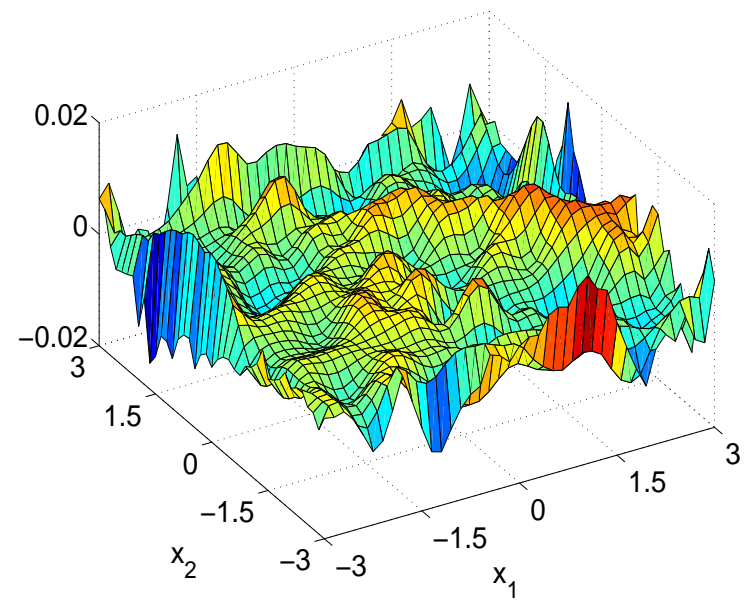

(c)

Figure 2: Example 1. (a) the true scatterer; (b) the reconstructed scatterer; (c) the difference between the true scatterer and the reconstructed one.

where $\tilde{q}$ is the reconstructed scatterer and $q$ is the true scatterer.

Example 1. Reconstruct a scatterer shown in Figure 2(a) using multiple frequency data. The initial guess is obtained from the Born approximation corresponding to weak scattering at low frequency. See Figure 3 for the relative error of reconstructions using different maximum wavenumber. It is clearly illustrated that the reconstruction is better using a large wavenumber than that using a smaller one. This result may be explained by Heisenberg's uncertainty principle. Figure 2(b) shows the reconstructed scatterer at wavenumber $\kappa=4.0$ and Figure $2(\mathrm{c})$ plots the difference between the true scatterer and the reconstructed one.

Example 2. Reconstruct a five-leave shape scatterer with a disc of radius 1 removed, see Figure 4(a). Figure 4(b) shows the initial guess from the MUSIC algorithm and Figure 4(c) shows the final reconstruction. The initial guesses are obtained via MUSIC algorithm and a level set representation 


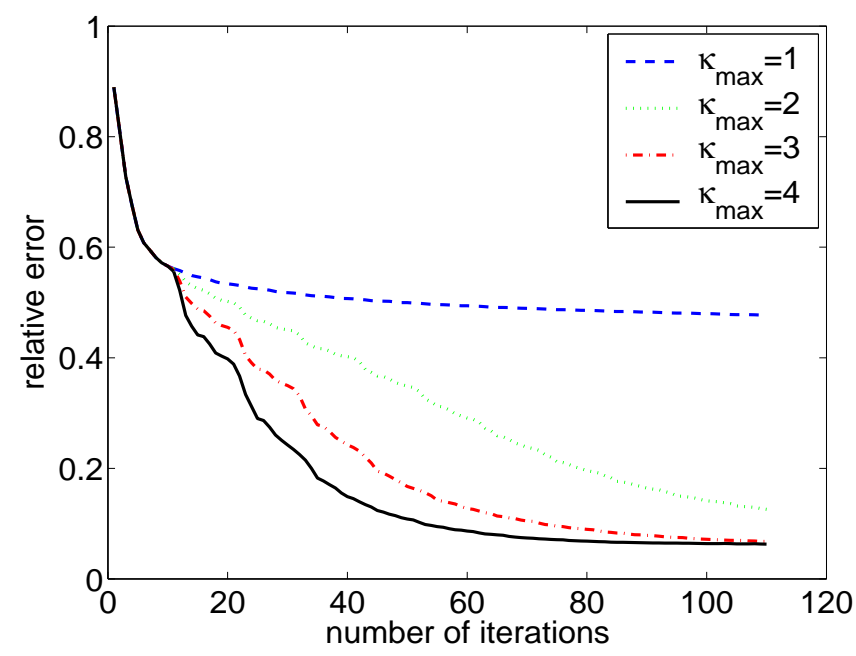

Figure 3: Example 1. The relative error of reconstructions.

at the wavenumber $\kappa=1$. The largest wavenumber used in the recursive linearization algorithm is $\kappa=6$. The step size for wavenumbers is 0.5 , i.e., the number of iteration along wavenumbers is 10 . From this example, we observe that the MUSIC algorithm does not provide detailed shape information from the starting low frequency data. However, it is a very fast direct algorithm to provide initial guesses. The final results after recursive linearization is very promising.

Example 3. Reconstruct a scatterer given in Figure 5(a) using single frequency data with an initial guess from the Born approximation corresponding to weak scattering at high spatial frequency. This scatterer is difficult to reconstruct because of the discontinuity across two circles. Figure 5(b) and Figure 5(c) respectively show the reconstructed scatterer and the difference between the true scatterer and the reconstructed using the wavenumber $\kappa=15$. The plots show that the error of the reconstruction occurs largely around the discontinuity, while the smooth part is recovered more accurately. As expected, the Gibbs phenomenon appears in the reconstructed scatterer near the discontinuity.

\section{Conclusion}

We have presented two regularized recursive linearization methods with respect to the wavenumber and the spatial frequency of a one-parameter family of plane waves. The recursive linearization algorithms are robust and efficient for solving the inverse medium scattering with multiple or single frequency. Finally, we point out some future directions along the line of this work. The first is concerned with the convergence analysis. Although our numerical experiments demonstrate the convergence and stability of the inversion algorithm, no rigorous mathematical analysis of the algorithms is available at present. Initial attempt has been made recently in [10] to establish convergence results by taking into account of the uncertainty principle. Another direction is to investigate inverse medium problems for Maxwell's equations with limited aperture case. An on-going effort of our group is to extend the approaches in this survey to the more complicated 3D model problems. 


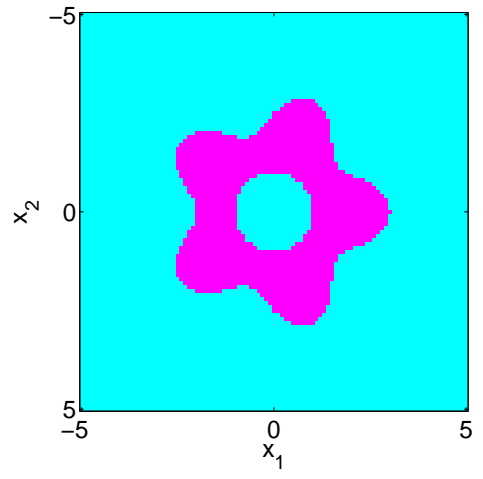

(a)

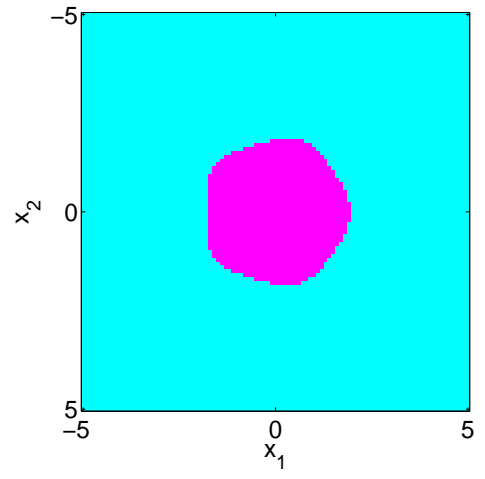

(b)

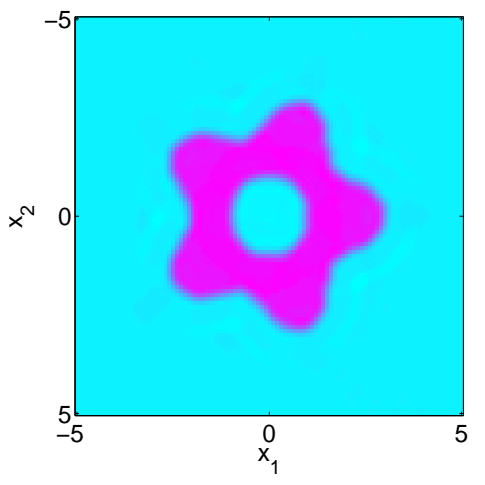

(c)

Figure 4: Example 2. (a) the true scatterer; (b) the initial guess; (c) the final reconstruction. 


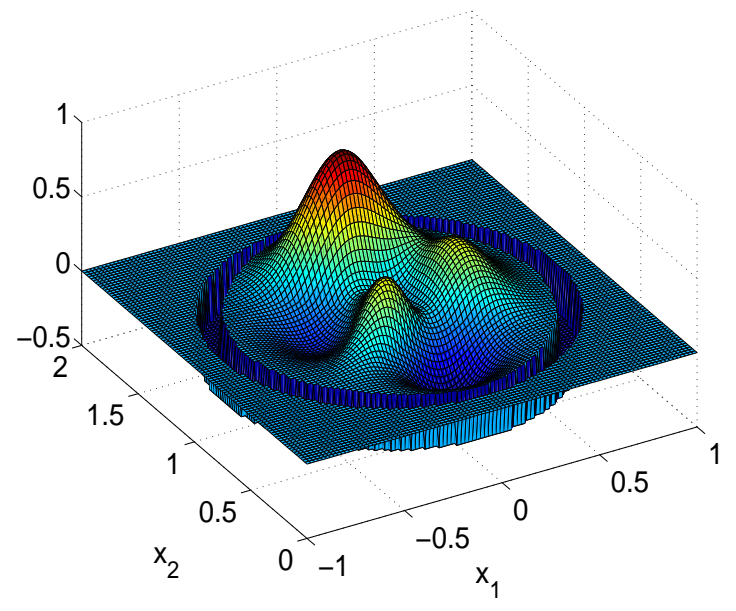

(a)

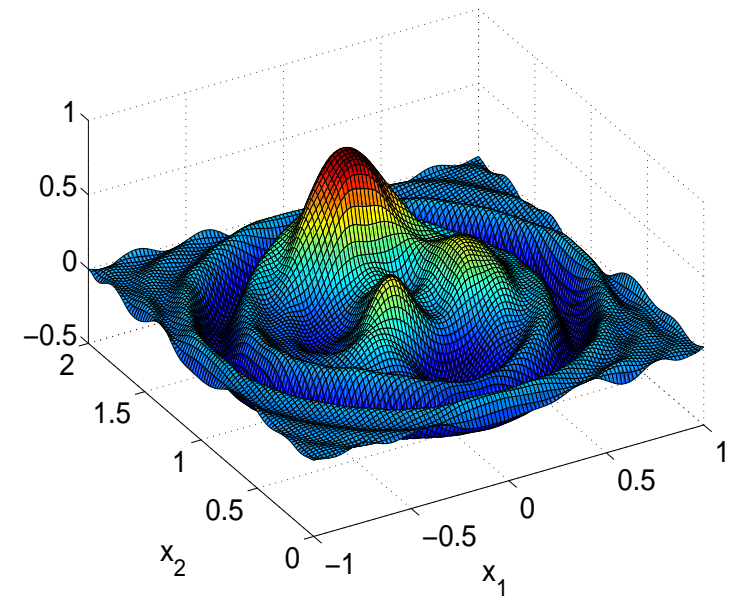

(b)

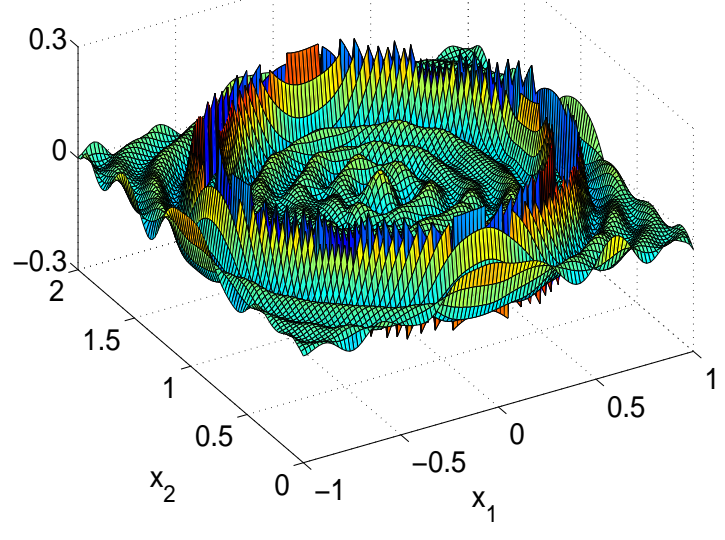

(c)

Figure 5: Example 3. (a) the true scatterer; (b) the reconstructed scatterer; (c) the difference between the true scatterer and the reconstructed one. 


\section{References}

[1] H. Ammari and J.-C. Nédélec, Low-frequency electromagnetic scattering, SIAM J. Math. Anal., 31 (2000), 836-861.

[2] H. Ammari and H. Kang, Reconstruction of Small Inhomogeneities from Boundary Measurements, Lecture Notes in Mathematics, Vol. 1846, Springer, N.Y., 2004.

[3] G. Bao, Y. Chen, and F. Ma, Regularity and stability for the scattering map of a linearized inverse medium problem, J. Math. Anal. Appl., 247 (2000), 255-271.

[4] G. Bao, S. Hou, and P. Li, Inverse scattering by a continuation method with initial guesses from a direct imaging algorithm, preprint.

[5] G. Bao and P. Li, Inverse medium scattering for the Helmholtz equation at fixed frequency, Inverse Problems, 21 (2005), 1621-1641.

[6] G. Bao and P. Li, Inverse medium scattering for three-dimensional time harmonic Maxwell equations, Inverse Problems, 20 (2004), L1-L7.

[7] G. Bao and P. Li, Inverse medium scattering problems for electromagnetic waves, SIAM J. Appl. Math., 65 (2005), 2049-1066.

[8] G. Bao and J. Liu, Numerical solution of inverse problems with multi-experimental limited aperture data, SIAM J. Sci. Comput., 25 (2003), 1102-1117.

[9] G. Bao and P. Li, Inverse medium scattering problem in near-field optics, J. Comput. Math., to appear.

[10] G. Bao and F. Triki, Error estimates for the recursive linearization of inverse medium problems, preprint.

[11] P. Carney and J. Schotland, Three-dimensional total internal reflection microscopy, Opt. Lett., 26 (2001), 1072-1074.

[12] V.Caselles, F.Catte, T.Coll, and F.Dibos, A geometric model for active contours in image processing, Numer. Math, 66 (1993), 1-31.

[13] V.Casseles, R. Kimmel, and G. Sapiro, On geodesic active contours, Int. J. Comput. Vis., 22 (1997), 61-79.

[14] Y. Chen, Inverse scattering via Heisenberg uncertainty principle, Inverse Problems, 13 (1997), 253-282.

[15] Y. Chen, Inverse scattering via skin effect, Inverse Problems, 13 (1997), 649-667.

[16] D. Courjon and C. Bainier, Near field microscopy and near field optics, Rep. Prog. Phys., 57 (1994), 989-1028. 
[17] D. Colton, J. Coyle, and P. Monk, Recent development in inverse acoustic scattering theory, SIAM Review, 42 (2000), 369-414.

[18] D. Colton and R. Kress, Integral Equation Methods in Scattering Theory, Wiley, New York, 1983.

[19] D. Colton and R. Kress, Inverse Acoustic and Electromagnetic Scattering Theory, SpringerVerlag, Berlin, 1998.

[20] D. Courjon, Near-field Microscopy and Near-field Optics, Imperial College Press, 2003.

[21] O. Dorn, E. Miller, and C. Rappaport, A shape reconstruction method for electromagnetic tomography using adjoint fields and level sets, Inverse Problems, 16 (2000), 1119-1156.

[22] H. Engl, M. Hanke, and A. Neubauer, Regularization of Inverse Problems, Dordrecht: Kluwer, 1996.

[23] D. Gilbarg and N. Trudinger, Elliptic Partial Differential Equations of Second Order, SpringerVerlag, New York, 1983.

[24] C. Girard and A. Dereux, Near-field optics theories, Rep. Prog. Phys., 59 (1996), 657-699.

[25] F. K. Gruber, E. A. Marengo, and A. J. Devaney, Time-reversal imaging with multiple signal classification considering multiple scattering between the targets, J. Acoust. Soc. Am., 115 (2004), $3042-3047$.

[26] T. Hohage, On the numerical solution of a three-dimensional inverse medium scattering problem, Inverse Problems, 17 (2001), 891-906.

[27] S. Hou, K. Solna, and H. Zhao, A direct imaging algorithm for extended targets, Inverse Problems, 22 (2006), 1151-1178.

[28] K. Ito, K. Kunisch, and Z. Li, Level-set function approach to an inverse interface problem, Inverse Problems, 17 (2001), 1225-1242.

[29] D. Jerison and C. Kenig, Unique continuation and absence of positive eigenvalues for Schrödinger operators, Ann. Math., 121 (1985), 463-488.

[30] J. Jin, The Finite Element Methods in Electromagnetics, John Wiley \& Sons, 2002.

[31] M. Kass, A. Witkin, and D. Terzopoulos, Snakes: Active contour models, Int. J. Comput. Vis., 1 (1988), 321-331.

[32] P. Monk, Finite Element Methods for Maxwell's Equations, Oxford University Press, Oxford, 2003.

[33] F. Natterer, The Mathematics of Computerized Tomography, Stuttgart: Teubner, 1986.

[34] F. Natterer and F. Wübbeling, A propagation-backpropagation method for ultrasound tomography, Inverse Problems, 11 (1995), 1225-1232. 
[35] J. Nédélec, Acoustic and Electromagnetic Equations: Integral Representations for Harmonic Problems, Springer, New York, 2000.

[36] S. Osher and J. Sethian, Fronts propagating with curvature-dependent speed: algorithms based on Hamilton-Jacobi formulations, J. Comput. Phys., 79 (1988), 12-49.

[37] D. Peng, B. Merriman, S. Osher, H. Zhao, and M. Kang, A PDE-based fast local level set method, J. Comput. Phys., 155(2) (1999), 410-438.

[38] M. Sussman, P. Smereka, and S. Osher, A level set approach for computing solutions to incompressible two-phase flows, J. Comput. Phys., 119 (1994), 146-159.

[39] M. Vögeler, Reconstruction of the three-dimensional refractive index in electromagnetic scattering by using a propagating-backpropagation method, Inverse Problems, 19 (2003), 739-753.

[40] H. Zhao, T. Chan, B. Merriman, and S. Osher, A variational level set approach to multiphase motion, J. Comput. Phys., 127 (1996), 179-195. 